\title{
Printed circuit board integrated fluxgate sensor
}

\author{
O. Dezuari * , E. Belloy, S.E. Gilbert, M.A.M. Gijs \\ Institute of Microsystems, Swiss Federal Institute of Technology (EPFL), CH-1015 Lausanne, Switzerland
}

\begin{abstract}
We have developed a cheap and simple trilayer printed circuit board (PCB)-based technology, adapted for the fabrication of fluxgate magnetic sensors. The two outer layers of the PCB stack comprise the electrical windings of the fluxgate, while the inner layer is made of patterned amorphous magnetic core with extremely high relative magnetic permeability $\left(\mu_{\mathrm{r}} \approx 100,000\right)$. The output voltage and the sensitivity of the fluxgate devices have been studied as a function of the external field and of the geometry of the magnetic core. We have found a relatively high sensitivity of $18 \mathrm{~V} / \mathrm{T}$ at an excitation current frequency of $10 \mathrm{kHz}$. The results obtained clearly show the potential of this miniaturised fluxgate device for application as a magnetic field sensor. (C) 2000 Elsevier Science S.A. All rights reserved.
\end{abstract}

Keywords: Fluxgate sensor; Sensitivity; High permeability; Compass; Vitrovac ${ }^{\circledR}$; Printed circuit board (PCB)

\section{Introduction}

In recent years, many magnetic sensing devices have been dramatically reduced in size, thanks to the advanced development of microfabrication technologies. An example of such device for the sensitive detection of magnetic fields is the magnetic fluxgate sensor [1-3]. A fluxgate consists of a magnetic bulk or ribbon core and electrical windings for the realisation of the excitation and detection coils around the magnetic core. Recently, several fluxgate sensors have been demonstrated, which were directly integrated on Si-wafers. In these applications, the magnetic material, which is at the heart of the fluxgate operation, is deposited using a vacuum deposition method or by electroplating technologies on $\mathrm{Si}$ [4-8].

In contrast to previous work based on $\mathrm{Si}$ microfabrication technology, we propose a new and inexpensive hybrid printed circuit board (PCB)-like technology for the realisation of fluxgate magnetic sensors [9]. The fluxgate devices are built up of three layers of which the outer layers carry the $\mathrm{Cu}$ winding coil patterns and the inner layer is a patterned high permeability ferromagnetic sheet core with a thickness of $25 \mu \mathrm{m}$. For this work, a particular alloy, known under the trade name Vitrovac ${ }^{\circledR} 6025$, (composi- tion $\left.(\mathrm{Co}, \mathrm{Fe}, \mathrm{Mo})_{73}(\mathrm{Si}, \mathrm{B})_{27}\right)$, was chosen primarily because of its extremely high relative permeability $\left(\mu_{\mathrm{r}} \approx 100,000\right)$ [10]. Both magnetic metal and copper layers are patterned using standard lithographic techniques. In between the magnetic metal and copper layers, there is a foil of solid epoxy glue (Prepreg ${ }^{\circledR}$ typically $100 \mu \mathrm{m}$ ) for insulation and assembly. Such material is well known in the assembly of multilayer PCB structures. Connection between the outer copper patterns is realised by electroplating methods to complete the windings. These fluxgate devices are about $600 \mu \mathrm{m}$ thick, with lateral dimensions of approximately 1 $\mathrm{cm}$.

\section{Fluxgate design}

Fig. 1 shows a design diagram for a fluxgate sensor with rectangularly shaped magnetic core. Two excitation coils of five windings each are symmetrically positioned around the inner detection coil (also with five windings). All windings are around the inner magnetic part of the structure; flux closure of the magnetic circuit is obtained via the two outer 'legs' of the magnetic core. Moreover, we have introduced two air gaps (of length $L_{\mathrm{g}}$ ) in the magnetic core. Gap lengths $L_{\mathrm{g}}$ have been varied between 0 and $1 \mathrm{~mm}$, to investigate their influence on the sensitivity and the magnetic field detection range. A photograph of a finished rectangular fluxgate sensor is displayed in Fig. 2. 


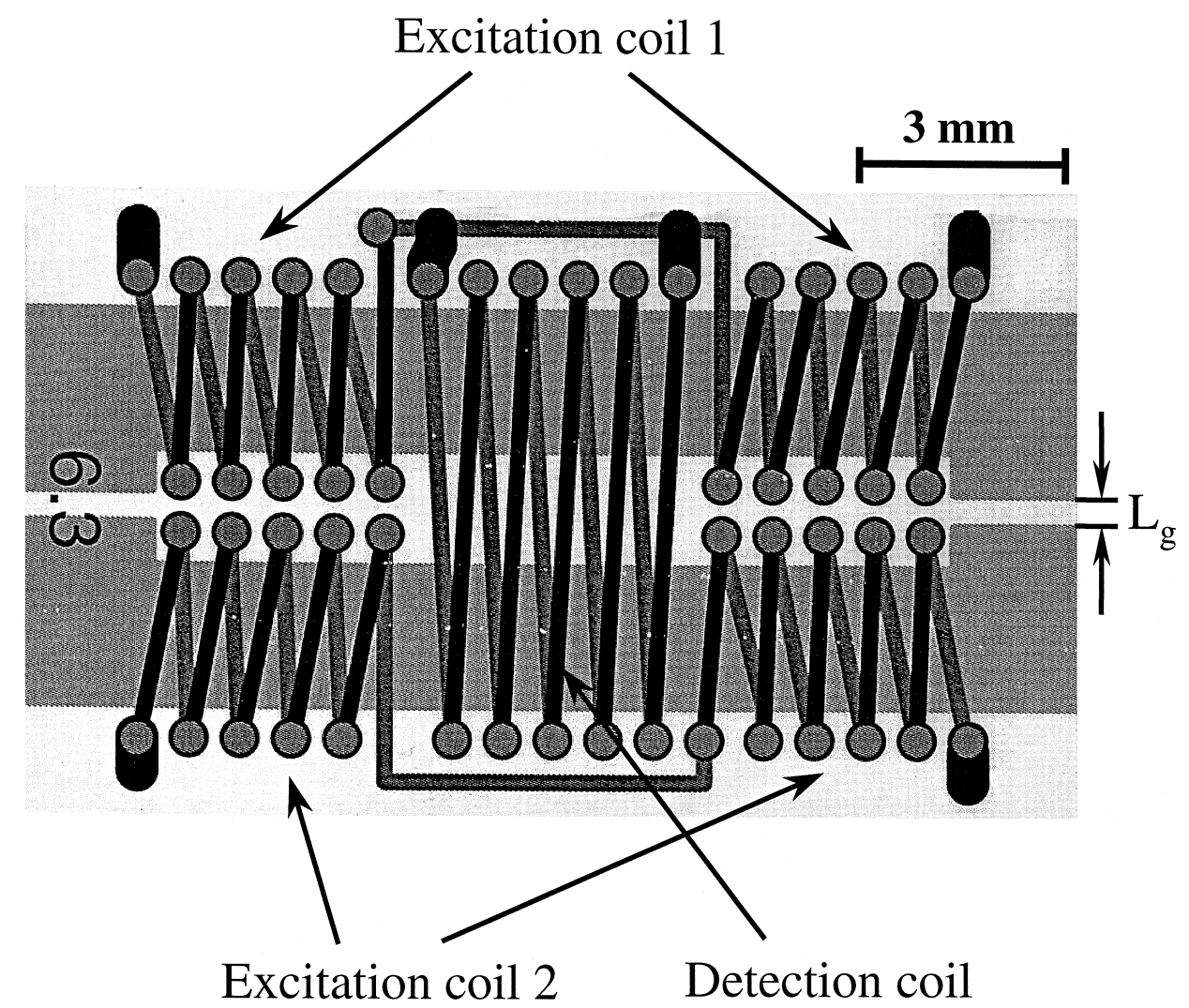

Fig. 1. Schematic diagram of a fluxgate sensor with rectangularly shaped magnetic material showing air gaps with length $L_{\mathrm{g}}$.

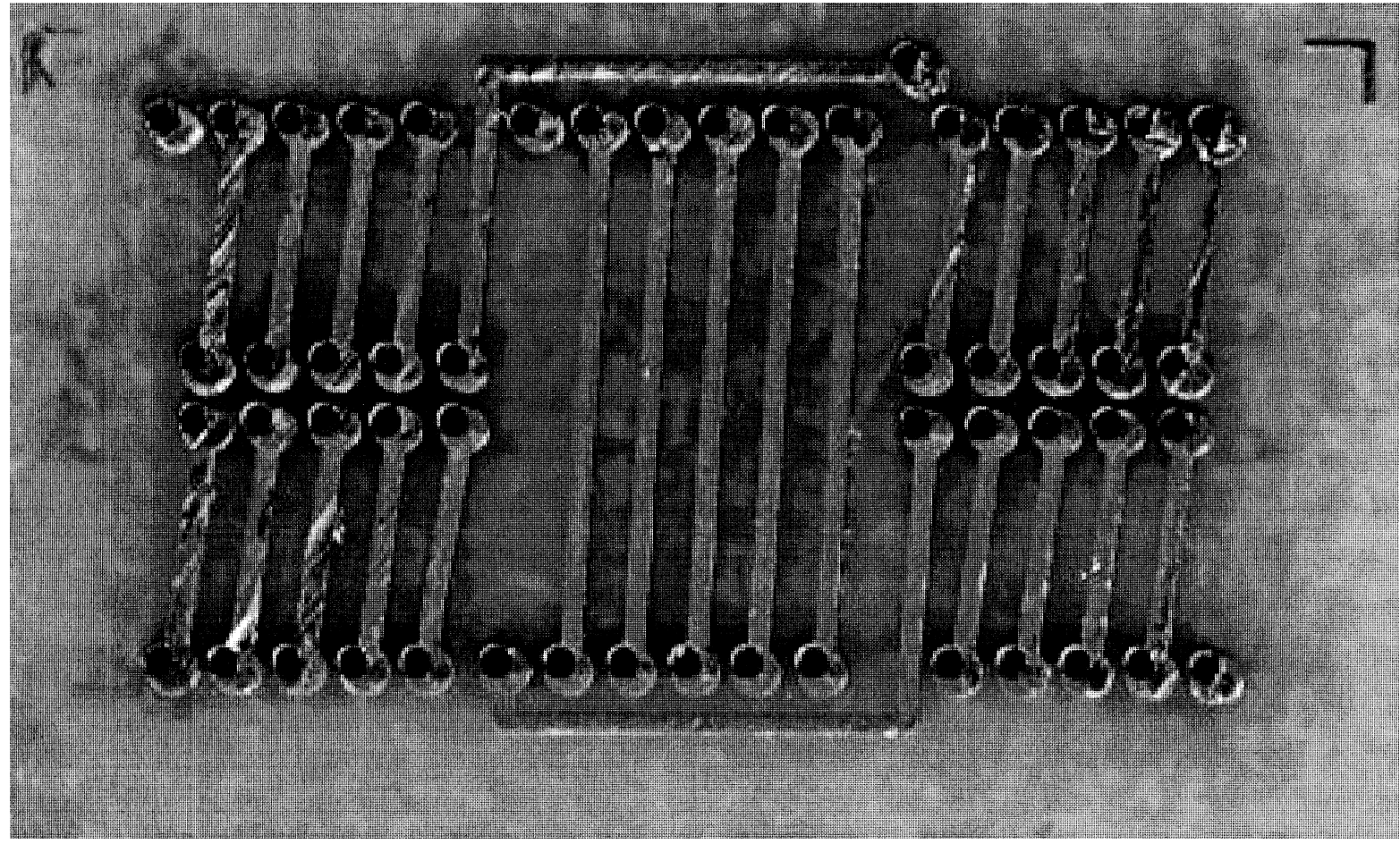

Fig. 2. Photograph of a finished rectangular fluxgate sensor. 


\section{Experimental results and discussion}

The principle of a fluxgate is based on the external magnetic field dependent periodical saturation of the ferromagnetic material. The combined action of the external field (to be measured) and excitation coils, driving the ferromagnetic core in saturation, leads to the generation of higher order harmonics of the fundamental excitation frequency in a detection coil surrounding the magnetic core. The second harmonic of the output can be measured using a lock-in technique, and is proportional to the external field for a certain field range (typically $0.01-100 \mu \mathrm{T}$ ).

\subsection{Dependence on the air gap}

Fig. 3a represents the second harmonic voltage $V_{2 \mathrm{f}}$ of the detection coil as a function of the external field $B_{\text {ext }}$ for various gap lengths $L_{\mathrm{g}}$, and at a frequency of $10 \mathrm{kHz}$ for an excitation current $I_{\mathrm{e}}$ of $300 \mathrm{~mA}_{p-p} . B_{\text {ext }}$ is applied in-plane perpendicular to the detection coil, as shown

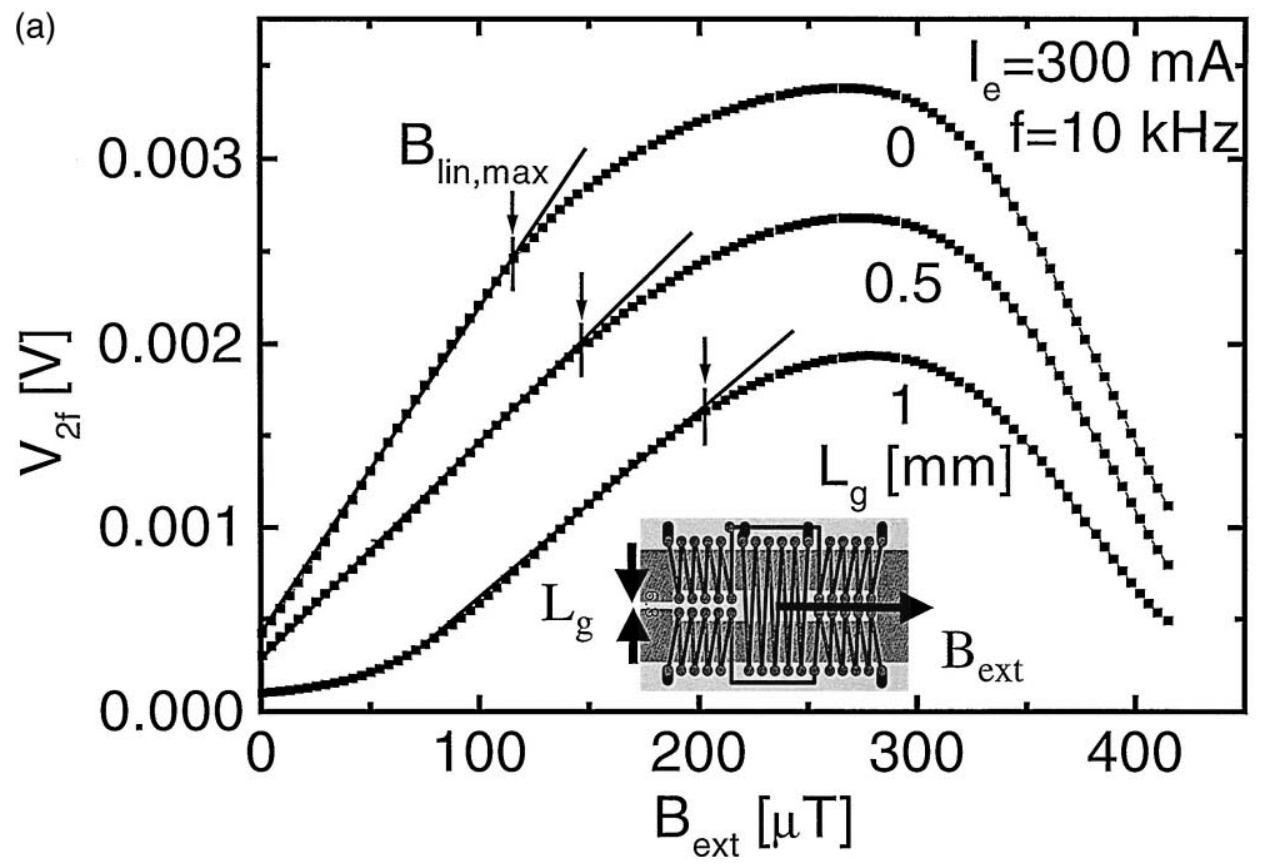

(b)

Fig. 3. (a) Second harmonic voltage $V_{2 \mathrm{f}}$ of the detection coil of a rectangular fluxgate sensor as a function of $B_{\text {ext }}$ for various gap length $L_{\mathrm{g}}$ at a frequency $f=10 \mathrm{kHz}$ and for an excitation current $I_{\mathrm{e}}$ of $300 \mathrm{~mA}$. (b) Sensitivity $S_{B}=\left(\mathrm{d} V_{2 \mathrm{f}}\right) /\left(\mathrm{d} B_{\text {ext }}\right)$ and maximum of the linear range $B_{\text {lin,max }}$, determined from the curves of a). 


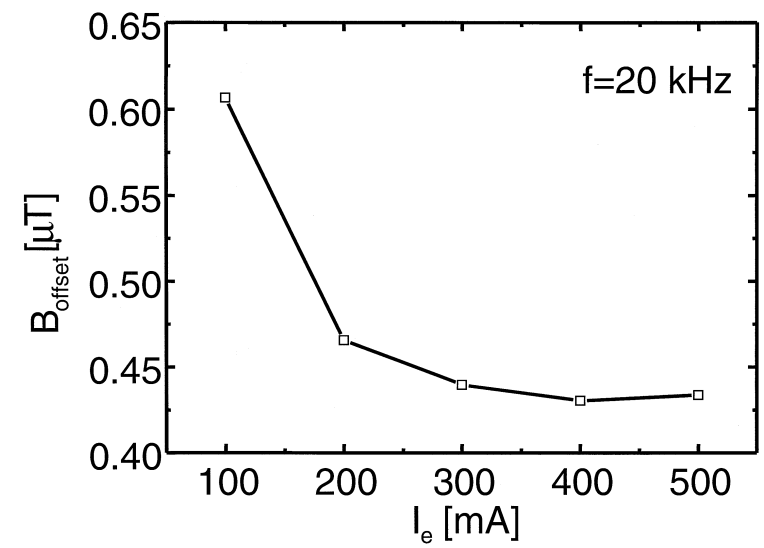

Fig. 4. Magnetic offset $B_{\text {offset }}$ of the fluxgate sensor as a function of excitation current $I_{\mathrm{e}}$ at $20 \mathrm{kHz}$, for the rectangular fluxgate without gap.

schematically by the arrow in the insert of Fig. 3a. The three curves are characterised by a linear slope at small $B_{\text {ext }}$. For $L_{\mathrm{g}}=0 \mathrm{~mm}$, one notes a limiting field for this linear range $\left(B_{\text {lin,max }}\right)$ of $110 \mu \mathrm{T}$, while the maximum value of $V_{2 \mathrm{f}}$ is around $280 \mu \mathrm{V}$. For $L_{\mathrm{g}}=0.5$ and $1 \mathrm{~mm}$, the maxima of the linear ranges $B_{\text {lin,max }}$ are shifted to higher values, while the maximum of the curves is around the same external field $B_{\text {ext }}=280 \mu \mathrm{T}$. Hence, the slopes of the linear parts of the $V_{2 \mathrm{f}}-B_{\text {ext }}$ curves decrease with increasing gap length $L_{\mathrm{g}}$. This graph clearly shows that, by choosing a certain air gap $L_{g}$, one can select the fluxgate sensor's sensitivity and its linear magnetic field range.

Fig. $3 \mathrm{~b}$ shows the sensitivity $S_{B}=\left(\mathrm{d} V_{2 \mathrm{f}}\right) /\left(\mathrm{d} B_{\text {ext }}\right)$ and the maximum of the linear range $B_{\mathrm{lin}, \max }$, determined from the curves of Fig. 3a. One notes a decrease of the sensitivity $S_{B}$ and an increase of the maximum of the linear range $B_{\text {lin,max }}$ with increasing gap length $L_{\mathrm{g}}$. In fact, $S_{B}$ decreases with $L_{\mathrm{g}}$. It has been established previously that $S_{B} \sim \mu_{4}^{*}$ [4] where $\mu_{\mathrm{r}}^{*}$ is the effective relative permeability decreasing with $L_{\mathrm{g}}$. By choosing a certain air gap $L_{\mathrm{g}}$, it hence is possible to make a compromise between the sensitivity and the magnetic field detection range.

\subsection{Magnetic offset of the fluxgate sensor}

To evaluate the minimum detection limit of our fluxgate sensing devices, we have performed measurements of the magnetic offset of the fluxgate sensor $B_{\text {offset }}$. We have measured the voltage $V_{2 \mathrm{f}}$ for a rectangular fluxgate, when cycling $B_{\text {ext }}$ from zero to saturation and back to zero. From the value of $V_{2 \mathrm{f}}$ at this point, one can calculate the offset by using the fluxgate's measured sensitivity curves. The results of such procedure are shown in Fig. 4 as a function of excitation current $I_{\mathrm{e}}$ at $20 \mathrm{kHz}$. One observes a decreasing $B_{\text {offset }}$, i.e., an improved minimum field detection limit, with increasing $I_{\mathrm{e}}$. The reason for this behaviour has to be sought in the better defined magnetic saturation state at high applied $I_{\mathrm{e}}$ (smaller width of magnetic hysteresis curve at high $I_{\mathrm{e}}$ ). The observed values of $B_{\text {offset }}$ for our fluxgate sensor clearly are well below the value of the earth's magnetic field, enabling application of our devices for example as a magnetic compass.

\section{Conclusions}

We have developed a cheap new hybrid PCB-based technology, for the realisation of fluxgate sensors with output characteristics that are equal to and even surpass those of previously reported devices. The $V_{2 \mathrm{f}}$ response of rectangular fluxgate sensors has been studied as a function of the gap length, the excitation current and excitation frequency. By appropriate selection of those parameters, one can select the fluxgate sensor's sensitivity and the linear magnetic field range. This new hybrid PCB/magnetic metal foil technology offers new possibilities for less expensive and facile realisation of high sensitivity fluxgate sensors.

\section{References}

[1] F. Primdahl, The fluxgate magnetometer, J. Phys. E: Sci. Instrum. 12 (1979) 241.

[2] P. Ripka, Review of magnetic fluxgate sensor, Sensors and Actuators A 33 (1992) 129.

[3] N. Pollock, Electronic compass using a fluxgate sensor, Wireless World, October, 1982, 49.

[4] S. Kawahito, Y. Sasaki, H. Sato, T. Nakamura, Y. Tadokoro, A fluxgate magnetic sensor with micro-solenoids and electroplated permalloy cores, Sensors and Actuators A 43 (1994) 128.

[5] T. Seitz, Fluxgate sensor in planar microtechnology, Sensors and Actuators A 21-23 (1990) 799.

[6] S.O. Choi, S. Kawahito, Y. Matsumoto, M. Ishida, Y. Tadokoro, An integrated micro fluxgate magnetic sensor, Sensors and Actuators A 55 (1996) 121.

[7] S. Kawahito, H. Satoh, M. Sutoh, Y. Tadokoro, High-resolution micro-fluxgate sensing elements using closely coupled coil structures, Sensors and Actuators A 54 (1996) 612.

[8] R. Gottfried-Gottfried, W. Budde, R. Jähne, H. Kück, B. Sauer, S. Ulbricht, U. Wende, A miniaturised magnetic-field sensor system consisting of a planar fluxgate sensor and a CMOS readout circuitry, Sensors and Actuators A 54 (1996) 443.

[9] O. Dezuari, E. Belloy, S.E. Gilbert, M.A.M. Gijs, A new hybrid technology for planar fluxgate sensor fabrication, submitted to IEEE Trans. Magn. (1998).

[10] Vitrovac ${ }^{\circledR}$ product literature, Vacuumschmelze, Hanau, Germany. 